\title{
Exploring the Limits of Quantum Nonlocality with Entangled Photons
}

\author{
Bradley G. Christensen, ${ }^{1, *}$ Yeong-Cherng Liang, ${ }^{2,3}$ Nicolas Brunner, ${ }^{4}$ Nicolas Gisin, ${ }^{5}$ and Paul G. Kwiat ${ }^{1}$ \\ ${ }^{1}$ Department of Physics, University of Illinois at Urbana-Champaign, Urbana, Illinois 61801, USA \\ ${ }^{2}$ Department of Physics, National Cheng Kung University, Tainan 701, Taiwan \\ ${ }^{3}$ Institute for Theoretical Physics, ETH Zürich, 8093 Zurich, Switzerland \\ ${ }^{4}$ Département de Physique Théorique, Université de Genève, 1211 Genève, Switzerland \\ ${ }^{5}$ Group of Applied Physics, University of Geneva, CH-1211 Geneva 4, Switzerland
}

(Received 16 July 2015; published 30 December 2015)

\begin{abstract}
Nonlocality is arguably among the most counterintuitive phenomena predicted by quantum theory. In recent years, the development of an abstract theory of nonlocality has brought a much deeper understanding of the subject, revealing a rich and complex phenomenon. In the current work, we present a systematic experimental exploration of the limits of quantum nonlocality. Using a versatile and high-fidelity source of pairs of polarization-entangled photons, we explore the boundary of quantum correlations, demonstrate the counterintuitive effect of more nonlocality with less entanglement, present the most nonlocal correlations ever reported, and achieve quantum correlations requiring the use of complex qubits. All of our results are in remarkable agreement with quantum predictions, and thus represent a thorough test of quantum theory. Pursuing such an approach is nevertheless highly desirable, as any deviation may provide evidence of new physics beyond the quantum model.
\end{abstract}

DOI: 10.1103/PhysRevX.5.041052

\section{INTRODUCTION}

Distant observers sharing a well-prepared entangled state can establish correlations that cannot be explained by any theory compatible with a natural notion of locality, as witnessed via a suitable Bell inequality violation [1]. Once viewed as marginal, nonlocality is today considered as one of the most fundamental aspects of quantum theory [2,3] and represents a powerful resource in quantum information science, in particular, in the context of the deviceindependent approach [4-6]. Experimental evidence is overwhelming, all major loopholes have been individually addressed [7-10], and three experiments closing both the locality and detection loopholes were just reported [11-13].

From a more abstract perspective, recent years have been marked by several developments providing a much deeper understanding of the phenomenon of quantum nonlocality. A generalized theory of nonlocality $[2,14,15]$ was developed, aimed at characterizing correlations satisfying the nosignaling principle (hence, not in direct conflict with relativity). Importantly, there exist no-signaling correlations - the most notable example being the highly nonlocal box of Popescu-Rohrlich [14] - that are stronger than any correlations realizable in quantum theory. Characterizing

\footnotetext{
* Corresponding author. bgchris2@illinois.edu

Published by the American Physical Society under the terms of the Creative Commons Attribution 3.0 License. Further distribution of this work must maintain attribution to the author(s) and the published article's title, journal citation, and DOI.
}

Subject Areas: Quantum Physics, Quantum Information

the boundary of quantum correlations (i.e., the separation from more general no-signaling correlations) is an important area of research today [16-18]. Intense theoretical research effort is also devoted to explain why superquantum-correlations are unlikely to exist in nature [19-25]. These ideas have clear potential to deepen our understanding of the foundations of quantum theory (see, e.g., Ref. [26] for a recent review), and may give a first glimpse of physics beyond the quantum model [27].

Another fundamental issue is the relation between entanglement and nonlocality. While early work showed that the two concepts are genuinely different, it was shown recently that entanglement and nonlocality are in fact not even monotonically related. Hence, it is possible to obtain "more nonlocality with less entanglement" not only at the qubit level [28] but also when there is no restriction on the size of the entanglement [29-31]: a strikingly counterintuitive effect. In particular, there exist portions of the boundary of quantum correlations that can only be accessed using weakly entangled states [29,31]; i.e., these correlations are provably impossible to reach using maximally entangled states of whatever Hilbert space dimension. Perhaps even more surprisingly, very weakly entangled states can lead to strongly nonlocal correlations, stronger still than the highly nonlocal PR box [32].

Although the above ideas triggered considerable attention from the theoretical community, they remain essentially unexplored at the experimental level. Indeed, most Bell experiments performed thus far [9-11,33-36] focus on the simplest (and most famous) Clauser-Horne-ShimonyHolt (CHSH) Bell inequality [37]; note that few 
exploratory works considered Bell tests in the multipartite setting [38-40] or for high-dimensional systems [41-43].

The goal of the present work is to start a systematic experimental exploration of the limits of quantum nonlocality. Using a high-quality entangled-photon source, we perform a wide range of Bell tests. In particular, we probe the boundary of quantum correlations in the simplest Bell scenario. We demonstrate the phenomenon of more nonlocality with less entanglement; specifically, using weakly entangled states, we observe (i) nonlocal correlations that could provably not have been obtained from any finitedimensional maximally entangled state and (ii) nonlocal correlations that could not have been obtained using a single PR box. Moreover, we observe the most nonlocal correlations ever reported, i.e., featuring the smallest local content [44], and provide the strongest bounds to date on the outcome predictability in a general class of physical theories consistent with the no-signaling principle [45]. Finally, we observe nonlocal correlations that certify the use of complex qubit measurements [46].

These results provide the most comprehensive experimental study of quantum nonlocality performed so far. All of our results are in remarkable agreement with quantum predictions. Nevertheless, we believe that pursuing such a large-scale exploration is of prime importance. These tests provide stringent verifications of quantum predictions, as any deviation could indicate new physics beyond quantum theory.

\section{CONCEPTS AND NOTATIONS}

First, we introduce the concepts and notations for generalized Bell tests, and then present the experiments. Consider two separated observers, Alice and Bob, performing local measurements on a shared quantum state $\rho$. Alice's choice of measurement settings is denoted by $x$ and the measurement outcome by $a$. Similarly, Bob's choice of measurement is denoted by $y$ and its outcome by $b$. The experiment is thus characterized by the joint distribution

$$
p(a, b \mid x, y)=\operatorname{Tr}\left(\rho M_{a \mid x} \otimes M_{b \mid y}\right),
$$

where $M_{a \mid x}\left(M_{b \mid y}\right)$ represents the measurement operators of Alice (Bob); see Fig. 1(a). In his seminal work, Bell introduced a natural concept of locality, which assumes that the local measurement outcomes depend on only a preestablished strategy and the choice of local measurements [1]. Specifically, a distribution is said to be local if it admits a decomposition of the form

$$
p(a, b \mid x, y)=\int d \lambda q(\lambda) p(a \mid x, \lambda) p(b \mid y, \lambda),
$$

where $\lambda$ denotes a shared local (hidden) variable, distributed according to the density $q(\lambda)$, and Alice's probability


FIG. 1. (a) Bell test scenario. Alice and Bob perform "black box" measurements on a shared (quantum) state $\rho$. The experiment is characterized by the data $\{p(a, b \mid x, y)\}$, i.e., a set of conditional probabilities for each pair of measurement outputs ( $a$ and $b$ ) given measurement settings $x$ and $y$. Based on the data $p(a, b \mid x, y)$, Bell inequalities [see Eq. (3)] can be tested. (b) Geometrical representation of nonsignaling correlations. The set of local $(\mathcal{L})$, quantum $(\mathcal{Q})$, and nonsignaling $(\mathcal{N S})$ distributions are projected onto a plane, where the following inclusion relations are clear: $\mathcal{L} \subset \mathcal{Q} \subset \mathcal{N S}$.

distribution-once $\lambda$ is given-is notably independent of Bob's input and output (and vice versa). For a given number of settings and outcomes the set of local distributions forms a polytope $\mathcal{L}$, the facets of which correspond to Bell inequalities [2]. These inequalities can be written as

$$
\mathcal{S}=\sum_{a, b, x, y} \beta_{a, b, x, y} p(a, b \mid x, y) \stackrel{\mathcal{L}}{\leq} L,
$$

where $\beta_{a, b, x, y}$ are integer coefficients and $L$ denotes the local bound of the inequality - the maximum of the quantity $\mathcal{S}$ over distributions from $\mathcal{L}$, i.e., of the form Eq. (2).

By performing judiciously chosen local measurements on an entangled quantum state, one can obtain distributions [Eq. (1)] that violate one (or more) Bell inequalities, and hence do not admit a decomposition of the form Eq. (2). Therefore, the set of quantum correlations $\mathcal{Q}$, i.e., those admitting a decomposition of the form Eq. (1), is strictly larger than the local set $\mathcal{L}$. Characterizing the quantum set $\mathcal{Q}$, or equivalently the limits of quantum nonlocality, turns out to be a hard problem [17,18]. In their seminal work, Popescu and Rohrlich [14] asked whether the principle of no-signaling (or relativistic causality) could be used to derive the limits of $\mathcal{Q}$ and surprisingly found this not to be the case. Specifically, they proved the existence of nosignaling correlations that are not achievable in quantum theory, the so-called "PR box" correlations. Therefore, the set of no-signaling correlations, denoted by $\mathcal{N S}$, is strictly larger than $\mathcal{Q}$, and we get the relation $\mathcal{L} \subset \mathcal{Q} \subset \mathcal{N S}$ [see Fig. 1(b)]. The study and characterization of the boundary between $\mathcal{Q}$ and $\mathcal{N S}$ is today a hot topic of research [26]. A central question is whether the limits of quantum nonlocality could be recovered from a simple physical 
principle (i.e., is it possible to derive quantum mechanics from just causality and another axiom?).

\section{EXPERIMENTAL SETUP}

Here, we experimentally explore the limits of quantum nonlocality using a high-quality source of entangled photons [7]. Our entanglement source consists of a 355$\mathrm{nm}$ pulsed laser focused onto two orthogonal nonlinear $\mathrm{BiBO}$ crystals to produce polarization-entangled photon pairs at $710 \mathrm{~nm}$, via spontaneous parametric downconversion: the first (second) crystal has an amplitude to create horizontal (vertical) polarized photon pairs, which interfere to produce the entangled state [47] (see Fig. 2). Using wave plates to control the polarization of the pump beam, we create polarization-entangled states with arbitrary degree of entanglement

$$
\left|\psi_{\theta}\right\rangle=\cos \theta|H, H\rangle+\sin \theta|V, V\rangle
$$

In addition to the ability to precisely tune the entangled state of the source, which is crucial for many of the Bell tests we perform, we also achieve extremely high state quality. To do so, we precompensate the temporal decoherence from group-velocity dispersion in the downconversion crystals with a BBO crystal [48], resulting in an interference visibility of $0.997 \pm 0.0005$ in all bases. The high state quality (along with the capability of creating a state with nearly any degree of entanglement) allows us to

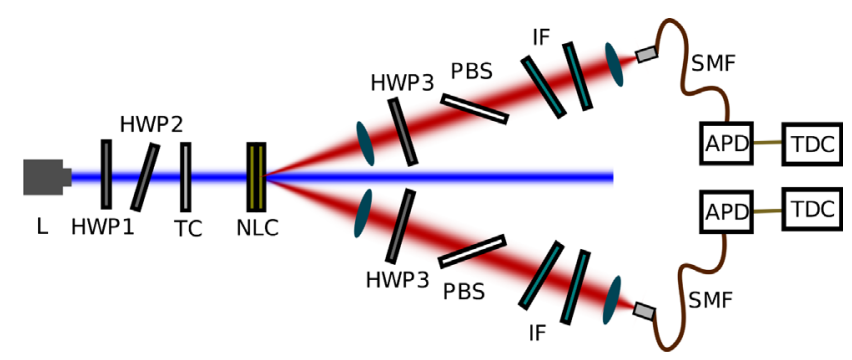

FIG. 2. A diagram of the entanglement source. The high-power laser (L) is prepared in a specific polarization state (depending on the Bell test) by two half-wave plates (HWP1 and HWP2). We precompensate for the temporal decoherence (arising from the group velocity dispersion in the down-conversion crystals) by passing the laser through a crystal (TC) designed to have the opposite group velocity dispersion. Passing the pump through a pair of orthogonal nonlinear crystals (NLC) produces the entangled photons. The measurements are performed using a motorized half-wave plate (HWP3) and a polarizing beam splitter (PBS). We then spectrally filter (IF) the photons to limit the collected bandwidth to $20 \mathrm{~nm}$, as well as spatially filter the photons using a single-mode fiber (SMF) to remove any spatial decoherence. Finally, the photons are detected using avalanche photodiodes (APD), the events of which are recorded on a timeto-digital converter (TDC) and saved on a computer for analysis. make measurements very close to the quantum mechanical bound in a large array of different Bell tests.

For the Bell tests, the local polarization measurements are implemented using a fixed Brewster-angle polarizing beam splitter, preceded by an adjustable half-wave plate, and followed by single-photon detectors to detect the transmitted photons. This allows for the implementation of arbitrary projective measurements of the polarization, represented by operators $A=\vec{a} \cdot \vec{\sigma}$ and $B=\vec{b} \cdot \vec{\sigma}$, where $\vec{a}$ and $\vec{b}$ are the Bloch vectors and $\vec{\sigma}=\left(\sigma_{x}, \sigma_{y}, \sigma_{z}\right)$ denotes the vector of Pauli matrices. Measurement outcomes are denoted by $a= \pm 1$ and $b= \pm 1$, where in our experiments the -1 outcome is measured by projecting onto the orthogonal polarization. To remove any potential systematic loopholes (e.g., seemingly better results due to laser power fluctuations), we measure each Bell inequality multiple times, where the measurements settings are applied in a different randomized order each time. Finally, to ensure the validity of the results, we do not perform any postprocessing of the data (e.g., accidental subtraction).

\section{EXPERIMENTS AND RESULTS}

We start our investigation by considering the simplest Bell scenario, featuring two binary measurements each for Alice and Bob. The set of local correlations, i.e., of the form Eq. (2), is fully captured by the CHSH inequality [37]:

$$
\mathcal{S}_{\mathrm{CHSH}}=E_{11}+E_{12}+E_{21}-E_{22} \stackrel{\mathcal{L}}{\leq 2},
$$

where $E_{x y} \equiv p(a=b \mid x, y)-p(a \neq b \mid x, y)$ denotes the correlation function. Quantum correlations can violate the above inequality up to $\mathcal{S}_{\mathrm{CHSH}}=2 \sqrt{2}$, the so-called Tsirelson bound [16]. More generally, quantum correlations must also satisfy the following family of inequalities:

$$
\mathcal{S}_{\mathrm{CHSH}} \cos \theta+\mathcal{S}_{\mathrm{CHSH}}^{\prime} \sin \theta \stackrel{\mathcal{Q}}{\leq} 2 \sqrt{2},
$$

parametrized by $\theta \in[0,2 \pi]$, and where $\mathcal{S}_{\mathrm{CHSH}}^{\prime}=-E_{11}+$ $E_{12}+E_{21}+E_{22}$ is a different representation (or symmetry) of the CHSH expression. Notably, the above quantum Bell inequalities are tight, in the sense that quantum correlations can achieve $2 \sqrt{2}$ for any $\theta \in[0,2 \pi]$. Specifically, inequality Eq. (6) can be saturated by performing appropriate local measurements on a maximally entangled state $\left|\psi_{\pi / 4}\right\rangle$ (see Appendix A). Therefore, the set of quantum correlations $\mathcal{Q}$ forms a circle (of radius $2 \sqrt{2}$ ) in the plane defined by $\mathcal{S}_{\mathrm{CHSH}}$ and $\mathcal{S}_{\mathrm{CHSH}}^{\prime}$. Figure 3 presents the experimental results that confirm these theoretical predictions with high accuracy. To make the measurements, we keep the entangled state fixed and vary the settings for 180 different values of $\theta$. The average radius of our measurements is 2.817 , with 8 data points falling beyond the limit of 

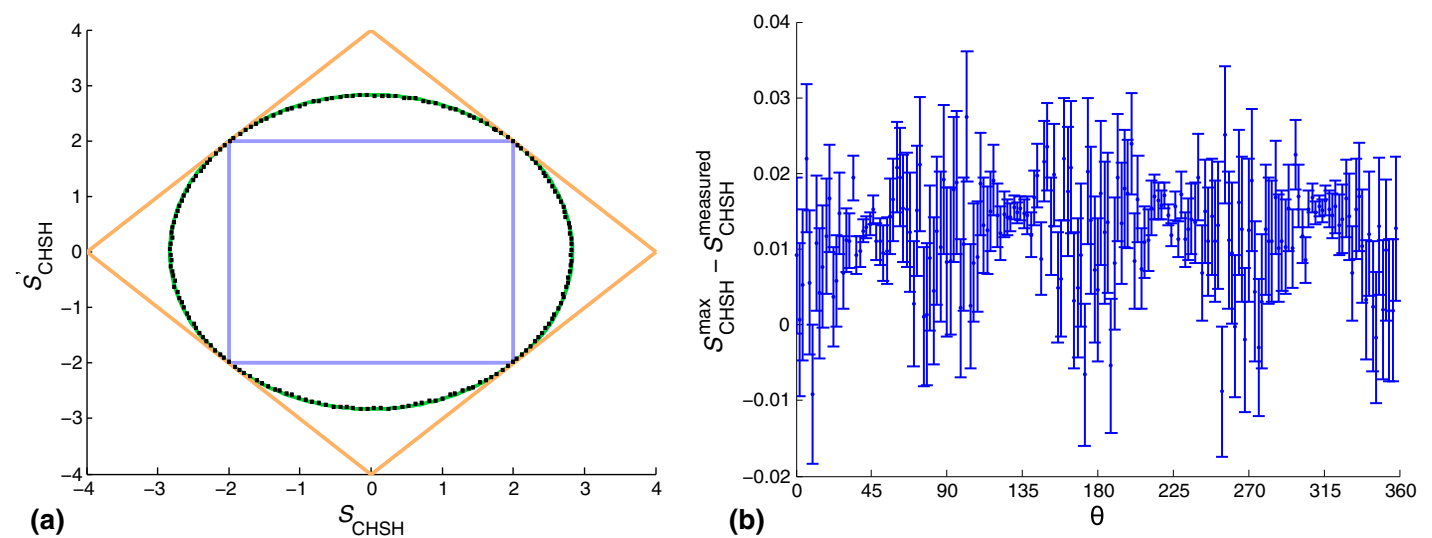

FIG. 3. Testing the boundary of quantum correlations in the simplest Bell test scenario. (a) Plot of the experimental measurements of the curve Eq. (6). Here, local correlations $(\mathcal{L})$ form the inner blue square, no-signaling correlations form the outer orange square (the vertices represent the PR box and its symmetries), and quantum correlations form the green circle; the black dots are the 180 measured data points, all of whose error bars lie within the thickness of the dot. (b) A comparison of the analyzed data with the quantum mechanical maximum $(2 \sqrt{2}$ ). Here, $\theta$ is defined in Eq. (6) and corresponds to rotating around the circle in Fig. 3(a). The vertical axis is the distance from $2 \sqrt{2}$ of the radius of curve formed by $\mathcal{S}_{\mathrm{CHSH}}^{\prime}$ and $\mathcal{S}_{\mathrm{CHSH}}$ (i.e., the radius of the data point at a given $\theta$ ). Plotted values greater than zero correspond to measured values less than $2 \sqrt{2}$. Here, the uncertainty is smaller for measurements around the vertices of the local correlations (at $45^{\circ}, 135^{\circ}, 225^{\circ}$, and $315^{\circ}$ ) due to large correlations between $\mathcal{S}_{\mathrm{CHSH}}^{\prime}$ and $\mathcal{S}_{\mathrm{CHSH}}$. We observe 8 data points beyond the quantum limit, which we expect to happen with a probability of 0.66 .

quantum mechanics. However, given our uncertainties of each point and the mean of our measurements, the probability of seeing 8 or more measurements beyond the quantum limit is 0.66 ; i.e., there is no conflict with quantum predictions.

It turns out, however, that the complete boundary of $\mathcal{Q}$ cannot be fully recovered by considering only maximally entangled states. That is, there exist sections of the nosignaling polytope where the quantum boundary can only be reached using partially entangled states. While studies $[28,49]$ on minimal detection efficiency required to close the detection loophole have already hinted at this for qubit entanglement, a more general observation with no restriction on the size of the entanglement has only been theoretically established recently in Refs. [29-31]. To demonstrate this bizarre phenomenon experimentally, we first strengthen the theoretical result obtained in Ref. [29].

Specifically, consider the projection plane defined by the parameters $\mathcal{S}_{\mathrm{CHSH}}$ and $-E_{1}^{A}-E_{1}^{B}$, where $E_{1}^{A}=$ $\sum_{a= \pm 1} a p(a \mid x=1)$ denotes Alice's marginal (similarly for Bob's marginal $\left.E_{1}^{B}\right)$. In order to find the quantum boundary in this plane, we consider the family of Bell inequalities

$$
\mathcal{S}_{\tau}=\mathcal{S}_{\mathrm{CHSH}}+2(1-\tau)\left[E_{1}^{A}+E_{1}^{B}\right] \stackrel{\mathcal{L}}{\leq} 2(2 \tau-1),
$$

with $1 \leq \tau \leq 3 / 2$. For $\tau=1$, we recover $\mathrm{CHSH}$, while for $1<\tau<3 / 2$, the inequality has the peculiar feature that the maximal quantum violation can only be obtained using partially entangled states [29]. Moreover, for $1 / \sqrt{2}+$ $1 / 2 \leq \tau \leq \frac{3}{2}$, as we show in Appendix B, the inequality cannot even be violated using any finite-size maximally entangled state. This illustrates the fact that weak entanglement can give rise to nonlocal correlations that cannot be reproduced using strong entanglement. We achieve violations of the above inequalities (for several values of the parameter $\tau$ ) extremely close to the theoretically predicted maximum, by adjusting the degree of entanglement and using the corresponding settings; see Fig. 4. For instance, tuning our source to produce weakly entangled states, we obtain clear violation of the inequality $\mathcal{S}_{\tau=1.300} \stackrel{\mathcal{L}}{\mathcal{L}} 3.2$, where we measure $\mathcal{S}_{\tau}=3.258 \pm 0.002$, which is impossible using maximally entangled states. Our results, thus, clearly illustrate the phenomenon of "more nonlocality with less entanglement" [29-31]. It is worth noting that, due to the small difference between the quantum maximum and the local bound $2(2 \tau-1)$, an experimental demonstration of the above phenomenon using inequality Eq. (7) is essentially impossible without extremely precise control of highquality weakly entangled states.

In the remainder of this paper, we go beyond the $\mathrm{CHSH}$ scenario and consider Bell inequalities featuring $n>2$ binary-outcome measurements per observer. This allows us to investigate other aspects of the phenomenon of quantum nonlocality. We start by considering the family of chained Bell inequalities [50,51]:

$$
\begin{aligned}
I_{n}= & \sum_{a, b= \pm 1}[p(a=b \mid n, 1)+p(a \neq b \mid n, n) \\
& \left.+\sum_{x=1}^{n-1} \sum_{y=x}^{x+1} p(a \neq b \mid x, y)\right] \geq 1
\end{aligned}
$$





FIG. 4. Testing the boundary of quantum correlations with the tilted Bell inequality. (a) A plot of the measured values for a projection where the quantum boundary can only be attained using partially entangled states. The orange line is the boundary for no-signaling correlations (the PR box sitting at the top), and the red line is the boundary of the set of correlations achievable by a maximally entangled state (see Appendix B), whereas the horizontal axis at $\mathcal{S}_{\mathrm{CHSH}}=2$ coincides with the boundary of the local set $\mathcal{L}$. The green curve represents the quantum boundary, with the black points corresponding to measured data points. For large values of $-E_{1}^{A}-E_{1}^{B}$ (corresponding to less entangled states), the system becomes increasingly sensitive to system noise (i.e., slight state creation and measurement imperfections), resulting in the measured values deviating slightly from the quantum curve. (b) A plot of the measured values for the tilted Bell inequalities. The red line is the bound of maximally entangled sates, the blue line is the local bound, and the black points are the analyzed data. The red and blue lines cross at $1 / \sqrt{2}+1 / 2$, where maximally entangled states can no longer violate a tilted Bell inequality. Here, for the measured points up to $\tau=1.323$, we see a value of $\mathcal{S}_{\tau}$ at least 3 standard deviations above the local bound; notably, we have violations for $\tau=1.223,1.250,1.265,1.296$, and 1.323 (circled data points in both plots), as well as $\tau=1.300$ (see text and Appendix C), none of which are possible for maximally entangled states in any dimension, implying that with less entanglement we have more nonlocality.

Using a maximally entangled state $\left|\psi_{\pi / 4}\right\rangle$, quantum theory allows one to obtain values up to $I_{n}=n[1-\cos (\pi / 2 n)]$. Note that, as $n$ increases, the quantum violation approaches the bound imposed by the no-signaling principle; namely, $I_{n}=0$ (here given by the algebraic minimum of $I_{n}$ ). Using our setup, we obtain violations of the chained inequality up to $n=45$. Because $I_{n}$ becomes increasingly sensitive to any noise in the system as $n$ increases, we find the strongest violation at $n=18$, with a value of $I_{18}=0.126 \pm 0.001$; see Fig. 5. For comparison, the previous best measurement of $I_{n}$ was $I_{7}=0.324 \pm 0.0027$ [52].

These violations have interesting consequences. First, they allow us to put strong lower bounds on the nonlocal content of the observed statistics $\mathbf{p}_{\mathrm{obs}}=\left\{p_{\mathrm{obs}}(a b \mid x y)\right\}$. Following the approach of Ref. [44], we can write the decomposition

$$
\mathbf{p}_{\mathrm{obs}}=(1-q) \mathbf{p}_{L}+q \mathbf{p}_{\mathrm{NS}}
$$

where $\mathbf{p}_{L}$ is a local distribution (inside $\mathcal{L}$ ) and $\mathbf{p}_{\mathrm{NS}}$ is a nosignaling distribution (achieved, e.g., via PR boxes), and then minimize $q \in[0,1]$ over any such decomposition. The minimal value $q_{\min }$ is then the nonlocal content of $\mathbf{p}_{\mathrm{obs}}$ and can be viewed as a measure of nonlocality. That is, we can think of $q_{\min }$ as being the likelihood that some nonlocal resource (e.g., a PR box) would need to be used in order to replicate the results. For an observed violation of the chained inequality, we can place a lower bound on the



FIG. 5. A plot of the measured chained Bell inequality values for $n=2$ to $n=45$. Here, the local limit is $I_{n}=1$ and the nosignaling limit is $I_{n}=0$. The quantum boundary in this case is the green line; our measured Bell inequality values are connected by the black line, with the error bars lying within the thickness of the line. The local content for a given $n$ is represented by distance from 0 to the measured $I_{n}$ value (black line), which is colored blue, and the nonlocal content is the distance from the measured value to 1 , colored orange. As the value of $I_{n}$ approaches 0 , the correlations present in the system match those of a PR box-if $I_{n}=0$ were measured, the system would require the use of a PR box for every measurement. Our measured points deviate from the quantum boundary due to the $0.3 \%$ noise from imperfect state preparation, which becomes more noticeable with larger number of measurements (e.g., $I_{45}$ requires 360 specific measurements along the Bloch sphere). 
nonlocal content: $q_{\min } \geq 1-I_{n}$ [53]. Notably, for the case $n=18$, we obtain $q_{\min }=0.874 \pm 0.001$, which represents the most nonlocal correlations ever produced experimentally. For comparison, the previous best bound was $q_{\min }=$ $0.782 \pm 0.014[54,55]$ (and if one maximally violates $S_{\mathrm{CHSH}}$, then $\left.q_{\min }=0.41\right)$.

Moreover, following the work of Ref. [45], we can place bounds on the outcome predictability in a general class of physical theories consistent with the no-signaling principle. While quantum theory predicts that the measurement results are fully random (e.g., one cannot predict locally which output port of the polarizing beam splitter each photon will be detected), there could be a super-quantumtheory that could predict better than quantum theory (that is, with a probability of success strictly greater than $1 / 2$ ) in which port each photon will be detected. This predictive power, represented by the probability $\delta$ of correctly guessing the output port, can be upper bounded from the observed violation of the chained Bell inequality. In our experiments, the best bound is obtained for the case $n=18$, for which we obtain $\delta=0.5702 \pm 0.0005$ (that is, given any possible extension of quantum theory satisfying the free-choice assumption [52], the measurement result could be guessed with a probability at most 57\%), which is the strongest experimental bound (closest to 50\%) to date; the previous bound was $\delta=0.6644 \pm 0.0014$ [52].

The above results on the chained Bell inequality show that, in order to reproduce the measured correlations, nonlocal resources (such as the PR box) must be used in more than $87 \%$ of the experimental rounds. While the chained Bell inequality provides an interesting metric of nonlocal content, there are, however, even more nonlocal correlations achievable using two-qubit entangled states, which can provably not be reproduced using a single PR box [32]. Interestingly, such correlations can arise only from partially entangled states, since maximally entangled states can always be perfectly simulated using a single PR box [56]. The accuracy of our experimental setup allows for the study of Bell inequalities that require the use of more than a single PR box. Specifically, consider the inequalities from Ref. [32] (for $n=3$ and $n=4$ ):

$$
\begin{aligned}
\mathcal{M}_{3322}= & E_{11}+E_{12}+E_{13}+E_{21}+E_{22}-E_{23} \\
& +E_{31}-E_{32}-E_{1}^{A}-E_{2}^{A}-E_{1}^{B}+E_{2}^{B} \stackrel{\mathcal{L}+1 \mathrm{PR}}{\leq} 6,
\end{aligned}
$$

$$
\begin{aligned}
\mathcal{M}_{4322}= & E_{11}+E_{12}+E_{13}+E_{21}-E_{23}+E_{24} \\
& +E_{31}-E_{32}-E_{34}-E_{1}^{A}-E_{2}^{A}-E_{3}^{A}-E_{1}^{B} \\
& \stackrel{\mathcal{L}+1 \mathrm{PR}}{\leq} 7,
\end{aligned}
$$

which cannot be violated by any local correlations supplemented by a single maximally nonlocal PR box $(\mathcal{L}+1 \mathrm{PR})$, which is viewed as a unit of nonlocality.
Nevertheless, by performing well-chosen measurements on a very weakly entangled state $\left(\left|\psi_{\approx 3 \pi / 7}\right\rangle\right)$, we observe violations of the above inequalities (see Table I). Note that since the observed statistics (leading to $\mathcal{M}_{3322}>6$ and $\mathcal{M}_{4322}>7$ ) could not have been obtained using a single PR box, they also cannot be obtained using a maximally entangled state $\left|\psi_{\pi / 4}\right\rangle$, and require the use of a weakly entangled state (or two PR boxes). Hence, we provide a second experimental verification of the phenomenon of more nonlocality with less entanglement.

Finally, we consider a Bell inequality that can certify the use of complex qubits (versus real qubits) [46]. Specifically, the Bell inequality is given by

$$
\begin{aligned}
\mathcal{S}_{E}= & E_{11}+E_{12}+E_{13}+E_{21}-E_{22}-E_{23} \\
& -E_{31}+E_{32}-E_{33}-E_{41}-E_{42}+E_{43} \stackrel{\mathcal{L}}{\leq} 6 .
\end{aligned}
$$

The optimal quantum violation is $\mathcal{S}_{E}=4 \sqrt{3} \simeq 6.928$, which can be obtained by using a maximally entangled two-qubit state $\left|\psi_{\pi / 4}\right\rangle$, and a set of highly symmetric qubit measurements. The measurements of Bob are given by three orthogonal vectors on the sphere, and Alice's measurements are given by the four vectors of the tetrahedron: $\vec{a}_{1}=(1 / \sqrt{3})(1,1,1), \quad \vec{a}_{2}=(1 / \sqrt{3})(1,-1,-1), \quad \vec{a}_{3}=$ $(1 / \sqrt{3})(-1,1,-1), \quad \vec{a}_{4}=(1 / \sqrt{3})(-1,-1,1)$ and $\vec{b}_{1}=$ $(1,0,0), \vec{b}_{2}=(0,1,0), \vec{b}_{3}=(0,0,1)$. To perform these measurements, we add an additional quarter-wave plate after HWP3 in Fig. 2. Implementing this strategy experimentally, we observe a violation of $\mathcal{S}_{E}=6.890 \pm 0.002$ close to the theoretical value. Interestingly, such a violation could not have been obtained using a real qubit strategy. Indeed, the use of measurement settings restricted to an equator of the Bloch sphere, i.e., real qubit measurements, can only provide violations up to $\mathcal{S}_{E}=2+2 \sqrt{5} \simeq 6.472$ [46]. Thus, the observed violation certifies the use of

TABLE I. A table of the measured values from two different Bell inequalities, $M_{3322}$ and $M_{4322}$, as defined in Eq. (10). For these inequalities, correlations from $\mathcal{L}$ and those augmented with the use of a single PR box (represented as $\mathcal{L}+1 \mathrm{PR}$ ) give rise to the same bound. Any measured values above the corresponding bound imply that the data are not only incompatible with Bell locality, but also with a single use of a PR box. Instead, two PR boxes must be used to replicate the data. The approximate quantum mechanical maximums (obtained using the tools of Refs. $[17,18,57,58])$ and the quantum mechanical maximums for two qubits are given as a reference.

\begin{tabular}{lcc}
\hline \hline Bell inequality & $\begin{array}{c}\text { Measured } \\
\text { value }\end{array}$ & $\begin{array}{c}\text { Quantum (two-qubit) } \\
\text { maximum }\end{array}$ \\
\hline$M_{3322} \stackrel{\mathcal{L}+1 \mathrm{PR}}{\leq} 6$ & $6.016 \pm 0.0003$ & $6.130(6.024)$ \\
$M_{4322} \stackrel{\mathcal{L}+1 \mathrm{PR}}{\leq} 7$ & $7.004 \pm 0.0004$ & $7.127(7.041)$ \\
\hline \hline
\end{tabular}


complex qubit measurements, i.e., spanning the Bloch sphere. Note, however, that any strategy involving complex qubit measurements can be mapped to an equivalent strategy involving two real qubits $[59,60]$.

\section{CONCLUSION}

To summarize, we report the observation of various facets of the rich phenomenon of quantum nonlocality. The results of our systematic experimental investigation of quantum nonlocal correlations are in extremely good agreement with quantum predictions; nevertheless, we believe that pursuing such tests is of significant value, as Bell inequalities are not only fundamental to quantum theory, but also can be used to discuss physics outside of the framework of quantum theory. By doing so, one can continue to place bounds on the features of theories beyond quantum mechanics, as we have here. Such continued experiments investigating the bounds of quantum theory are important, as any valid deviation with quantum predictions, e.g., by observing stronger correlations than predicted by quantum theory, would provide evidence of new physics beyond the quantum model. Furthermore, nonlocality has important applications towards quantum information protocols, though the optimal way to quantify the nonlocality present in a system is still an open question (see, e.g., Ref. [61]). Here, we experimentally verify, for the first time, that for certain correlations from nonmaximally entangled states, two PR boxes (i.e., two units of the nonlocal resource) are required to recreate the correlations from these weakly entangled states. A natural question then is if these systems could be used advantageously for certain quantum information tasks.

\section{ACKNOWLEDGMENTS}

This research was supported by NSF Grant No. PHY 1205870, the Ministry of Education, Taiwan, Republic of China, through "The Aim for the Top University Project" granted to the National Cheng Kung University (NCKU), the Swiss NCCR-QSIT, the Swiss National Science Foundation (Grant No. PP00P2_138917 and Starting Grant DIAQ), and SEFRI (COST action MP1006).

Note added.-Recently, a related work appeared, presenting the most precise measurement of the Tsirelson bound of the CHSH Bell inequality [62].

\section{APPENDIX A: CHARACTERIZATION OF THE QUANTUM BOUNDARY}

Here, we discuss in detail the characterization of the boundary of quantum correlations in a two-dimensional projection of the no-signaling polytope in the case of binary inputs and outputs (i.e., the CHSH scenario). Specifically, let us consider the two-dimensional plane (Fig. 3) defined by the expectation values of

$$
\begin{gathered}
\mathcal{S}_{\mathrm{CHSH}}=E_{11}+E_{12}+E_{21}-E_{22}, \\
\mathcal{S}_{\mathrm{CHSH}}^{\prime}=-E_{11}+E_{12}+E_{21}+E_{22} .
\end{gathered}
$$

Note that the correlation functions $E_{x y}$ can equivalently be seen as the average value of the product of \pm 1 outcome local (projective) measurements; i.e.,

$$
E_{x y}=\sum_{a, b= \pm 1} a b p(a, b \mid x, y) .
$$

They can thus be evaluated in quantum theory as $E_{x y}=\operatorname{Tr}\left(\rho A_{x} B_{y}\right)$, where $A_{x}, \quad B_{y}$ are dichotomic observables satisfying

$$
A_{x}^{2}=B_{y}^{2}=\mathbb{1}, \quad\left[A_{x}, B_{y}\right]=0 \quad \forall x, y .
$$

The boundary of the set of legitimate quantum distributions in this two-dimensional plane is given by the circle (see also Refs. [25,63]):

$$
\mathcal{S}_{\mathrm{CHSH}}^{2}+\mathcal{S}_{\mathrm{CHSH}}^{2} \leq 8,
$$

or, equivalently,

$\mathcal{S}_{\mathrm{CHSH}} \cos \theta+\mathcal{S}_{\mathrm{CHSH}}^{\prime} \sin \theta \leq 2 \sqrt{2} \quad \forall \theta \in[0,2 \pi]$.

To see that this is the case, let us note that for any dichotomic observables $A_{1}, A_{2}, \quad B_{1}, \quad B_{2}$ satisfying Eq. (A4), and any $\theta \in[0,2 \pi]$, the following identity holds true:

$$
\begin{aligned}
2 \sqrt{2} \mathbb{1}-\mathcal{B} & \\
= & \frac{1}{\sqrt{2}}\left[\sin \left(\frac{\pi}{4}+\theta\right) A_{2}+\cos \left(\frac{\pi}{4}+\theta\right) A_{1}-B_{1}\right]^{2} \\
& +\frac{1}{\sqrt{2}}\left[\cos \left(\frac{\pi}{4}+\theta\right) A_{2}-\sin \left(\frac{\pi}{4}+\theta\right) A_{1}+B_{2}\right]^{2},
\end{aligned}
$$

where $\mathcal{B}$ is the Bell operator [64] associated with the Bell expression given in the left-hand side of Eq. (A6); i.e.,

$$
\begin{aligned}
\mathcal{B}= & \sum_{x, y=1}^{2}\left\{\left[\cos \theta(-1)^{(x-1)(y-1)}+\sin \theta(-1)^{x y}\right] A_{x} B_{y}\right\} \\
= & \sqrt{2}\left[\sin \left(\frac{\pi}{4}+\theta\right)\left(A_{1} B_{2}+A_{2} B_{1}\right)\right. \\
& \left.+\cos \left(\frac{\pi}{4}+\theta\right)\left(A_{1} B_{1}-A_{2} B_{2}\right)\right] .
\end{aligned}
$$

Since the right-hand side of Eq. (A7) is a sum of nonnegative operators, it then follows that for any quantum 
state $\rho$, and hence for any quantum correlation, we must have

$2 \sqrt{2}-\operatorname{Tr}(\rho \mathcal{B})=2 \sqrt{2}-\left(\mathcal{S}_{\mathrm{CHSH}} \cos \theta+\mathcal{S}_{\mathrm{CHSH}}^{\prime} \sin \theta\right) \geq 0$.

The above bound on the set of quantum correlations is indeed achievable. Explicitly, for each $\theta$, by measuring the following observables,

$$
\begin{aligned}
& A_{1}=\sigma_{x}, \quad A_{2}=\sigma_{z}, \\
& B_{1}=-\sin \chi \sigma_{z}-\cos \chi \sigma_{x}, \quad B_{2}=\cos \chi \sigma_{z}-\sin \chi \sigma_{x},
\end{aligned}
$$

with $\chi=\theta-(3 \pi / 4)$ on the maximally entangled twoqubit state,

$$
\left|\psi_{\pi / 4}\right\rangle=\frac{1}{\sqrt{2}}(|H, H\rangle+|V, V\rangle)
$$

[with $|H\rangle(|V\rangle)$ being the +1 ( $(-1)$ eigenstate of $\sigma_{z}$ ], we arrive at quantum correlations that saturate the inequality given in Eq. (A6). By varying $\theta$ over the entire interval $[0,2 \pi]$, it can be verified that one indeed generates the entire (circular) boundary of the quantum set in this two-dimensional plane.

\section{APPENDIX B: UPPER-BOUNDING QUANTUM VIOLATION BY MAXIMALLY ENTANGLED STATES}

Let us denote by $\tau_{\mathrm{Cr}}=\frac{1}{2}+(1 / \sqrt{2}) \approx 1.2071$ and by $\left|\Phi_{d}^{+}\right\rangle$the maximally entangled state of local Hilbert space dimension $d$, i.e.,

$$
\left|\Phi_{d}^{+}\right\rangle=\frac{1}{\sqrt{d}} \sum_{i=0}^{d-1}|i\rangle|i\rangle,
$$

where $d \geq 2$. Here, we provide further details showing the following observation.

Observation 1: For $\tau \in\left[\tau_{\mathrm{Cr}}, \frac{3}{2}\right]$, the family of Bell inequalities given by Eq. (7) cannot be violated by any finite-dimensional maximally entangled state $\left|\Phi_{d}^{+}\right\rangle$.

Proof.-Let us first note that for arbitrary $\tau \in\left[\tau_{\mathrm{Cr}}, \frac{3}{2}\right]$, the Bell inequality $\mathcal{S}_{\tau}$ can be written as a convex combination of that for $\tau=\tau_{\mathrm{Cr}}$ and that for $\tau=\frac{3}{2}$. Moreover, for any given quantum state $\rho$ (and given experimental scenario), it is easy to show that the set of Bell inequalities satisfied by $\rho$ is a convex set. Since $\mathcal{S}_{\tau}$ for $\tau=\frac{3}{2}$ cannot be violated by any legitimate probability distribution [29], it suffices to show that $\mathcal{S}_{\tau}$ for $\tau=\tau_{\mathrm{Cr}}$ also cannot be violated by $\left|\Phi_{d}^{+}\right\rangle$(for any finite $d$ ).
To show that $n o$ finite-dimensional $\left|\Phi_{d}^{+}\right\rangle$can violate the Bell inequality $\mathcal{S}_{\tau}$ for $\tau=\tau_{\mathrm{Cr}}$, we make use of the hierarchy of semidefinite programs (SDPs) considered in Ref. [65] for characterizing exactly the quantum correlations achievable by $\left|\Phi_{d}^{+}\right\rangle$. Specifically, to obtain (an upper bound on) the maximal value of $\mathcal{S}_{\tau=\tau_{\mathrm{Cr}}}$ attainable by finite-dimensional $\left|\Phi_{d}^{+}\right\rangle$, it suffices to consider a fixed level of the hierarchy, and solve a SDP over the positive semidefinite (moment) matrix variable $\Gamma$ such that (1) certain entries of $\Gamma$ are required to be non-negative, (2) certain entries of $\Gamma$ are required to be identical, and (3) a particular entry of $\Gamma$ is required to be " 1 " (for details, see Ref. [65], pp. 16, 17).

To this end, we consider $\Gamma$ defined by the 17 symbolic operators $\mathbb{1}, A_{x}^{+}, B_{y}^{+}, A_{x}^{+} B_{y}^{+}, B_{y}^{+} A_{x}^{+}, B_{y}^{+} B_{y^{\prime}}^{+}, A_{x}^{+} A_{x^{\prime}}^{+}$, with $x$, $x^{\prime}, y, y^{\prime} \in 1,2$ and $x \neq x^{\prime}, y \neq y^{\prime}$. Solving the corresponding SDP via the solver SEDUMI (interfaced through YALMIP [66]), we find that the quantum value of $\mathcal{S}_{\tau=\tau_{\mathrm{Cr}}}$ attainable by any finite-dimensional $\left|\Phi_{d}^{+}\right\rangle$is upper bounded by $2\left(2 \tau_{\mathrm{Cr}}-1\right)+\epsilon$, with $\epsilon \approx 1.09 \times 10^{-8}$, which is vanishing within the numerical precision of the solver. In other words, after accounting for the numerical error present in the optimization problem, the output of the SDP provides a numerical certificate that no finite-dimensional maximally entangled state can violate the Bell inequality $\mathcal{S}_{\tau}$ for $\tau=\tau_{\mathrm{Cr}}$. This completes the proof of Observation 1 .

Note that as $\tau$ increases, the equality $\mathcal{S}_{\tau}=2(2 \tau-1)$, cf. Eq. (7), corresponds to a plane tilting from the horizontal axis towards the vertical axis at $-E_{1}^{A}-E_{1}^{B}=$ 2; see Fig. 4(a). For $\tau=\tau_{\mathrm{Cr}}$, this plane corresponds precisely to the red solid straight line joining the points $(0,2 \sqrt{2})$ and $(2,0)$. Observation 1 thus translates to the fact that, in Fig. 4(a), all correlations present in the region between the actual quantum boundary (green curve) and the red solid line are unattainable by finite-dimensional maximally entangled states - a fact that can also be independently verified by solving an analogous SDP that maximizes the value of $\mathcal{S}_{\mathrm{CHSH}}$ under the equality constraint that marginal correlations $-E_{1}^{A}-E_{1}^{B}$ take on specific values in the interval $[0,2]$.

Finally, let us note that the same numerical technique could also be used to show that both the $M_{3322}$ and the $M_{4322}$ inequality hold true (to within a numerical precision of $10^{-8}$ ) for all correlations arising from the maximally entangled state of any Hilbert space dimension.

\section{APPENDIX C: DETAILED DATA AND ESTIMATED STATES}

In this Appendix, we give the results of the analyzed data and quantum states used for each Bell test presented in this paper. First, for the data collected for the projection onto the $\mathcal{S}_{\mathrm{CHSH}} \cos \theta$ and $\mathcal{S}_{\mathrm{CHSH}}^{\prime} \sin \theta$ axes, we use maximally entangled states, altering the measurement settings to rotate around the circle in Fig. 3. Here, we collect data for $1 \mathrm{~s}$ at 
each setting (where each point requires 16 total measurement combinations). To calculate the likelihood, given our measurements, of having the observed 8 (or more) events beyond the quantum limit, first, we consider our expected radius to be the mean of all measured radii, calculated to be 2.817. Next, we assume the calculated error bars to correspond to one $\sigma$ from the mean (that is, each data point comes from a Gaussian distribution with a mean of the mean radius and a standard deviation given by the error bar). From here, we can compute the probability of each data point being measured greater than $2 \sqrt{2}$. We then assume that the probability distribution of seeing $k$ events beyond the quantum limit follows a Gaussian distribution with mean $\mu=\sum_{i} p_{i}=8.677$ and variance of $\sigma^{2}=$ $\sum_{i} p_{i}\left(1-p_{i}\right)=7.928$. This distribution then determines our probability of seeing 8 or more events of 0.66 .

For the plot of the tilted Bell inequality in Fig. 4 [Eq. (7)], we collect data for $15 \mathrm{~s}$ for each setting (again, 16 total measurement setting combinations). We use states of varying degree of entanglement, which we cite by listing the $\theta$ value in the state $\cos \theta|H, H\rangle+\sin \theta|V, V\rangle$. The analyzed data are displayed in Table II. As a note, the value in the text listed for $\mathcal{S}_{\tau=1.3}$ has separately optimized settings (instead of automatically generated settings), as well as is measured for $100 \mathrm{~s}$.

For the chained Bell inequality [Eq. (8)], $I_{n}$ is the chained Bell parameter, with $\nu_{n}$ being the measurement bias. The measurement bias is the deviation of Alice's (or

TABLE II. Analyzed data and estimated parameters for the tilted Bell inequality [Eq. (7)]. Here, the estimate of the uncertainty of $\mathcal{S}_{\tau}$ is given by $\Delta \mathcal{S}_{\tau}$.

\begin{tabular}{lcccccc}
\hline \hline$\tau$ & $\mathcal{S}_{\mathrm{CHSH}}$ & $-E_{1}-E_{2}$ & $\mathcal{S}_{\tau}$ & $\Delta \mathcal{S}_{\tau}$ & Local bound & $\theta$ \\
\hline 1.001 & 2.828 & 0.052 & 2.828 & 0.011 & 2.004 & 45.0 \\
1.020 & 2.827 & 0.120 & 2.837 & 0.010 & 2.080 & 46.5 \\
1.039 & 2.816 & 0.220 & 2.833 & 0.010 & 2.156 & 48.1 \\
1.063 & 2.800 & 0.320 & 2.840 & 0.010 & 2.252 & 49.8 \\
1.095 & 2.764 & 0.480 & 2.855 & 0.009 & 2.380 & 52.2 \\
1.128 & 2.736 & 0.620 & 2.895 & 0.009 & 2.512 & 54.9 \\
1.137 & 2.712 & 0.720 & 2.909 & 0.008 & 2.548 & 55.5 \\
1.171 & 2.660 & 0.860 & 2.954 & 0.008 & 2.684 & 58.5 \\
1.193 & 2.616 & 0.980 & 2.994 & 0.007 & 2.772 & 60.2 \\
1.223 & 2.564 & 1.120 & 3.064 & 0.007 & 2.892 & 62.7 \\
1.250 & 2.504 & 1.240 & 3.124 & 0.006 & 3.000 & 65.2 \\
1.265 & 2.456 & 1.320 & 3.156 & 0.006 & 3.060 & 66.4 \\
1.296 & 2.368 & 1.460 & 3.232 & 0.005 & 3.184 & 69.2 \\
1.323 & 2.304 & 1.580 & 3.325 & 0.005 & 3.292 & 71.7 \\
1.348 & 2.228 & 1.680 & 3.397 & 0.004 & 3.392 & 74.2 \\
1.369 & 2.168 & 1.760 & 3.467 & 0.003 & 3.476 & 76.3 \\
1.390 & 2.120 & 1.820 & 3.540 & 0.003 & 3.560 & 78.4 \\
1.410 & 2.064 & 1.880 & 3.606 & 0.002 & 3.640 & 80.5 \\
1.424 & 2.036 & 1.920 & 3.664 & 0.002 & 3.696 & 81.9 \\
1.435 & 2.016 & 1.932 & 3.697 & 0.002 & 3.740 & 82.9 \\
1.442 & 2.000 & 1.946 & 3.721 & 0.002 & 3.768 & 83.7 \\
1.449 & 1.964 & 1.957 & 3.722 & 0.002 & 3.796 & 84.6 \\
\hline \hline & & & & & & \\
\hline
\end{tabular}

Bob's) individual measurements from being completely random, that is, the difference in probability of measuring output -1 to measuring output 1 [calculated by $p(1 \mid x)-$ $p(-1 \mid x)]$. The bias given in Table III (and the bias used in calculating $\delta_{n}$ ) is the maximum bias over all possible measurement settings. Finally, $\delta_{n}$ is the bound on the predictive power, with $\Delta \delta_{n}$ being the uncertainty. The uncertainty of $I_{n}$ is approximately twice as large as the $\Delta \delta_{n}$ (since $\delta_{n} \propto I_{n} / 2$ ). For these measurements, we use a

TABLE III. Analyzed data for the chained Bell inequality [Eq. (8)].

\begin{tabular}{|c|c|c|c|c|}
\hline$\underline{n}$ & $I_{n}$ & $\nu_{n}$ & $\delta_{n}$ & $\Delta \delta_{n}$ \\
\hline 2 & 0.5931 & 0.0062 & 0.8028 & 0.0016 \\
\hline 3 & 0.4115 & 0.0058 & 0.7116 & 0.0014 \\
\hline 4 & 0.3148 & 0.0055 & 0.6629 & 0.0013 \\
\hline 5 & 0.2624 & 0.0068 & 0.6380 & 0.0012 \\
\hline 6 & 0.2230 & 0.0058 & 0.6173 & 0.0012 \\
\hline 7 & 0.1965 & 0.0065 & 0.6048 & 0.0011 \\
\hline 8 & 0.1812 & 0.0059 & 0.5964 & 0.0011 \\
\hline 9 & 0.1667 & 0.0073 & 0.5906 & 0.0011 \\
\hline 10 & 0.1539 & 0.0066 & 0.5836 & 0.0011 \\
\hline 11 & 0.1479 & 0.0069 & 0.5809 & 0.0011 \\
\hline 12 & 0.1419 & 0.0069 & 0.5778 & 0.0011 \\
\hline 13 & 0.1396 & 0.0065 & 0.5763 & 0.0011 \\
\hline 14 & 0.1357 & 0.0064 & 0.5742 & 0.0011 \\
\hline 15 & 0.1324 & 0.0077 & 0.5739 & 0.0010 \\
\hline 16 & 0.1312 & 0.0061 & 0.5718 & 0.0010 \\
\hline 17 & 0.1294 & 0.0064 & 0.5711 & 0.0010 \\
\hline 18 & 0.1262 & 0.0065 & 0.5702 & 0.0005 \\
\hline 19 & 0.1318 & 0.0070 & 0.5714 & 0.0005 \\
\hline 20 & 0.1290 & 0.0075 & 0.5722 & 0.0005 \\
\hline 21 & 0.1279 & 0.0074 & 0.5709 & 0.0005 \\
\hline 22 & 0.1291 & 0.0071 & 0.5717 & 0.0010 \\
\hline 23 & 0.1287 & 0.0065 & 0.5708 & 0.0010 \\
\hline 24 & 0.1325 & 0.0072 & 0.5734 & 0.0010 \\
\hline 25 & 0.1312 & 0.0074 & 0.5730 & 0.0010 \\
\hline 26 & 0.1380 & 0.0067 & 0.5757 & 0.0011 \\
\hline 27 & 0.1372 & 0.0070 & 0.5755 & 0.0010 \\
\hline 28 & 0.1389 & 0.0073 & 0.5768 & 0.0011 \\
\hline 29 & 0.1409 & 0.0073 & 0.5777 & 0.0011 \\
\hline 30 & 0.1429 & 0.0069 & 0.5783 & 0.0011 \\
\hline 31 & 0.1456 & 0.0075 & 0.5803 & 0.0011 \\
\hline 32 & 0.1474 & 0.0066 & 0.5803 & 0.0011 \\
\hline 33 & 0.1475 & 0.0070 & 0.5808 & 0.0011 \\
\hline 34 & 0.1506 & 0.0083 & 0.5836 & 0.0011 \\
\hline 35 & 0.1547 & 0.0073 & 0.5846 & 0.0011 \\
\hline 36 & 0.1573 & 0.0066 & 0.5853 & 0.0011 \\
\hline 37 & 0.1577 & 0.0081 & 0.5870 & 0.0011 \\
\hline 38 & 0.1594 & 0.0072 & 0.5869 & 0.0011 \\
\hline 39 & 0.1655 & 0.0072 & 0.5899 & 0.0011 \\
\hline 40 & 0.1665 & 0.0070 & 0.5903 & 0.0011 \\
\hline 41 & 0.1698 & 0.0073 & 0.5922 & 0.0011 \\
\hline 42 & 0.1716 & 0.0065 & 0.5923 & 0.0011 \\
\hline 43 & 0.1750 & 0.0067 & 0.5942 & 0.0011 \\
\hline 44 & 0.1810 & 0.0069 & 0.5974 & 0.0011 \\
\hline 45 & 0.1801 & 0.0079 & 0.5980 & 0.0011 \\
\hline
\end{tabular}


TABLE IV. Details for the $M_{3322}$ and $M_{4322}$ Bell inequalities [Eq. (10)].

\begin{tabular}{lcccccccc}
\hline \hline Inequality & $\theta$ & $a_{1}$ & $a_{2}$ & $a_{3}$ & $a_{4}$ & $b_{1}$ & $b_{2}$ & $b_{3}$ \\
\hline$M_{3322}$ & 77.2 & -1.2 & 27.2 & -35.2 & Not & applicable-0.7 & 9.2 & -20.3 \\
$M_{4322}$ & 76.6 & 0 & 61 & 45 & 119 & 15.6 & 164.3 & 0 \\
\hline \hline
\end{tabular}

maximally entangled state and collect data for $5 \mathrm{~s}$ at each measurement setting, except from $n=18$ to $n=21$, where we collect for $20 \mathrm{~s}$ at each setting, as the first scan through all values of $n$ shows the lowest value in that region. The analyzed data for the chained Bell inequality are shown in Table III.

Finally, in Table IV we list the measurement settings and states for the $M_{3322}$ and $M_{4322}$ Bell inequalities. The settings are given as the angle in the projection onto the state $\cos a_{i}|H\rangle+\sin a_{i}|V\rangle$ (and similarly for $b_{j}$ ). Here, data are collected data for $1200 \mathrm{~s}$ at each measurement setting.

[1] J. S. Bell, On the EPR Paradox, Physics 1, 195 (1964).

[2] N. Brunner, D. Cavalcanti, S. Pironio, V. Scarani, and S. Wehner, Bell Nonlocality, Rev. Mod. Phys. 86, 419 (2014).

[3] P. Shadbolt, J. Mathews, A. Laing, and J. O'Brien, Testing Foundations of Quantum Mechanics with Photons, Nat. Phys. 10, 278 (2014).

[4] A. Acin, N. Brunner, N. Gisin, S. Massar, S. Pironio, and V. Scarani, Device-Independent Security of Quantum Cryptography against Collective Attacks, Phys. Rev. Lett. 98, 230501 (2007).

[5] R. Colbeck, Ph.D. thesis, University of Cambridge, 2007.

[6] S. Pironio et al., Random Numbers Certified by Bell's Theorem, Nature (London) 464, 1021 (2010).

[7] B. G. Christensen et al., Detection-Loophole-Free Test of Quantum Nonlocality, and Applications, Phys. Rev. Lett. 111, 130406 (2013).

[8] M. Giustina et al., Bell Violation Using Entangled Photons without the Fair-Sampling Assumption, Nature (London) 497, 227 (2013).

[9] A. Aspect, J. Dalibard, and G. Roger, Experimental Test of Bell's Inequalities Using Time-Varying Analyzers, Phys. Rev. Lett. 49, 1804 (1982).

[10] M. A. Rowe, D. Kielpinski, V. Meyer, C. A. Sackett, W. M. Itano, C. Monroe, and D. G. Wineland, Experimental Violation of a Bell's Inequality with Efficient Detection, Nature (London) 409, 791 (2001).

[11] B. Hensen et al., Experimental Loophole-Free Violation of a Bell Inequality Using Entangled Electron Spins Separated by $1.3 \mathrm{~km}$, Nature (London) 526, 682 (2015).

[12] L. K. Shalm et al., Strong Loophole-Free Test of Local Realism, Phys. Rev. Lett. 115, 250402 (2015).

[13] M. Giustina et al., Significant-Loophole-Free Test of Bell's Theorem with Entangled Photons, Phys. Rev. Lett. 115, 250401 (2015).

[14] S. Popescu and D. Rohrlich, Causality and Non-Locality as Axioms for Quantum Mechanics, Found. Phys. 24, 379 (1994).
[15] J. Barrett, N. Linden, S. Massar, S. Pironio, S. Popescu, and D. Roberts, Nonlocal Correlations as an InformationTheoretic Resource, Phys. Rev. A 71, 022101 (2005).

[16] B. S. Tsirelson, Quantum Generalizations of Bell's Inequality, Lett. Math. Phys. 4, 93 (1980).

[17] M. Navascués, S. Pironio, and A. Acín, A Convergent Hierarchy of Semidefinite Programs Characterizing the Set of Quantum Correlations, New J. Phys. 10, 073013 (2008).

[18] A. C. Doherty, Y.-C. Liang, B. Toner, and S. Wehner, in Proceedings of the 23rd IEEE Conference on Computational Complexity (IEEE Computer Society, College Park, MD, 2008), pp. 199-210.

[19] W. van Dam, Implausible Consequences of Superstrong Nonlocality, arXiv:quant-ph/0501159.

[20] M. Pawlowski, T. Paterek, D. Kaszlikowski, V. Scarani, A. Winter, and M. Zukowski, Information Causality as a Physical Principle, Nature (London) 461, 1101 (2009).

[21] T. Fritz, A. B. Sainz, R. Augusiak, J. Bohr Brask, R. Chaves, A. Leverrier, and A. Acin, Local Orthogonality as a Multipartite Principle for Quantum Correlations, Nat. Commun. 4, 2263 (2013).

[22] M. Navascues and H. Wunderlich, A Glance beyond the Quantum Model, Proc. R. Soc. A 466, 881 (2010).

[23] D. Rohrlich, Quantum Theory: A Two Time Success Story (Springer, New York, 2013).

[24] N. Gisin, Quantum Measurement of Spins and Magnets, and the Classical Limit of PR-Boxes, arXiv:1407.8122.

[25] J. Allcock, N. Brunner, M. Pawlowski, and V. Scarani, Recovering Part of the Quantum Boundary from Information Causality, Phys. Rev. A 80, 040103(R) (2009).

[26] S. Popescu, Nonlocality beyond Quantum Mechanics, Nat. Phys. 10, 264 (2014).

[27] M. Navascués, Y. Guryanova, M. J. Hoban, and A. Acin, Almost Quantum Correlations, Nat. Commun. 6, 6288 (2015).

[28] P. H. Eberhard, Background Level and Counter Efficiencies Required for a Loophole-Free Einstein-Podolsky-Rosen Experiment, Phys. Rev. A 47, R747 (1993).

[29] Y.-C. Liang, T. Vértesi, and N. Brunner, SemiDevice-Independent Bounds on Entanglement, Phys. Rev. A 83, 022108 (2011).

[30] M. Junge and C. Palazuelos, Large Violation of Bell Inequalities with Low Entanglement, Commun. Math. Phys. 306, 695 (2011).

[31] T. Vidick and S. Wehner, More Nonlocality with Less Entanglement, Phys. Rev. A 83, 052310 (2011).

[32] N. Brunner, N. Gisin, and V. Scarani, Entanglement and Non-Locality Are Different Resources, New J. Phys. 7, 88 (2005).

[33] J. Hofmann, M. Krug, N. Ortegel, L. Gerard, M. Weber, W. Rosenfeld, and H. Weinfurter, Heralded Entanglement between Widely Separated Atoms, Science 337, 72 (2012).

[34] W. Tittel, J. Brendel, H. Zbinden, and N. Gisin, Violation of Bell Inequalities by Photons More Than $10 \mathrm{~km}$ Apart, Phys. Rev. Lett. 81, 3563 (1998).

[35] G. Weihs, T. Jennewein, C. Simon, H. Weinfurter, and A. Zeilinger, Violation of Bell's Inequality under Strict Einstein Locality Conditions, Phys. Rev. Lett. 81, 5039 (1998). 
[36] M. Ansmann et al., Violation of Bell's Inequality in Josephson Phase Qubits, Nature (London) 461, 504 (2009).

[37] J. F. Clauser, M. A. Horne, A. Shimony, and R. A. Holt, Proposed Experiment to Test Local Hidden-Variable Theories, Phys. Rev. Lett. 23, 880 (1969).

[38] J.-P. Pan, D. Bouwmeester, M. Daniell, H. Weinfurter, and A. Zeilinger, Experimental Test of Quantum Nonlocality in Three-Photon Greenberger-Horne-Zeilinger Entanglement, Nature (London) 403, 515 (2000).

[39] J. Lavoie, R. Kaltenbaek, and K. Resch, Experimental Violation of Svetlichny's Inequality, New J. Phys. 11, 073051 (2009).

[40] C. Erven et al., Experimental Three-Photon Quantum Nonlocality under Strict Locality Conditions, Nat. Photonics 8, 292 (2014).

[41] A. Mair, A. Vaziri, G. Weihs, and A. Zeilinger, Entanglement of the Orbital Angular Momentum States of Photons, Nature (London) 412, 313 (2001).

[42] R. T. Thew, A. Acin, H. Zbinden, and N. Gisin, Bell-Type Test of Energy-Time Entangled Qutrits, Phys. Rev. Lett. 93, 010503 (2004).

[43] A. C. Dada, J. Leach, G. S. Buller, M. J. Padgett, and E. Andersson, Experimental High-Dimensional Two-Photon Entanglement and Violations of Generalized Bell Inequalities, Nat. Phys. 7, 677 (2011).

[44] A. Elitzur, S. Popescu, and D. Rohrlich, Quantum Nonlocality for Each Pair in an Ensemble, Phys. Lett. A 162, 25 (1992).

[45] R. Colbeck and R. Renner, No Extension of Quantum Theory Can Have Improved Predictive Power, Nat. Commun. 2, 411 (2011).

[46] N. Gisin, Bell Inequalities: Many Questions, A Few Answers, arXiv:quant-ph/0702021; in Essays in Honour of A. Shimony, edited by W. C. Myrvold and J. Christian, The Western Ontario Series in Philosophy of Science (Springer, New York, 2009), pp. 125-140.

[47] P. G. Kwiat, E. Waks, A. G. White, I. Appelbaum, and P. H. Eberhard, Ultrabright Source of Polarization-Entangled Photons, Phys. Rev. A 60, R773 (1999).

[48] R. Rangarajan, M. Goggin, and P. G. Kwiat, Optimizing Type-I Polarization-Entangled Photons, Opt. Express 17, 18920 (2009).

[49] G. Vallone, G. Lima, E. S. Gómez, G. Canãs, J.-Å. Larsson, P. Mataloni, and A. Cabello, Bell Scenarios in which Nonlocality and Entanglement Are Inversely Related, Phys. Rev. A 89, 012102 (2014).

[50] P. Pearle, Hidden-Variable Example Based upon Data Rejection, Phys. Rev. D 2, 1418 (1970).
[51] S. Braunstein and C. Caves, Wringing out Better Bell Inequalities, Ann. Phys. (N.Y.) 202, 22 (1990).

[52] T. E. Stuart, J. A. Slater, R. Colbeck, R. Renner, and W. Tittel, Experimental Bound on the Maximum Predictive Power of Physical Theories, Phys. Rev. Lett. 109, 020402 (2012).

[53] J. Barrett, A. Kent, and S. Pironio, Maximally Nonlocal and Monogamous Quantum Correlations, Phys. Rev. Lett. 97, 170409 (2006).

[54] L. Aolita, R. Gallego, A. Acin, A. Chiuri, G. Vallone, P. Mataloni, and A. Cabello, Fully Nonlocal Quantum Correlations, Phys. Rev. A 85, 032107 (2012).

[55] T. Yang, Q. Zhang, J. Zhang, J. Yin, Z. Zhao, M. Zukowski, Z.-B. Chen, and J.-W. Pan, All-versus-Nothing Violation of Local Realism by Two-Photon, Four-Dimensional Entanglement, Phys. Rev. Lett. 95, 240406 (2005).

[56] N. J. Cerf, N. Gisin, S. Massar, and S. Popescu, Simulating Maximal Quantum Entanglement without Communication, Phys. Rev. Lett. 94, 220403 (2005).

[57] Y.-C. Liang and A.C. Doherty, Bounds on Quantum Correlations in Bell-Inequality Experiments, Phys. Rev. A 75, 042103 (2007).

[58] Y.-C. Liang, C. W. Lim, and D. L. Deng, Reexamination of a Multisetting Bell Inequality for Qudits, Phys. Rev. A 80, 052116 (2009).

[59] M. McKague, M. Mosca, and N. Gisin, Simulating Quantum Systems Using Real Hilbert Spaces, Phys. Rev. Lett. 102, 020505 (2009).

[60] K. F. Pál and T. Vértesi, Efficiency of Higher-Dimensional Hilbert Spaces for the Violation of Bell Inequalities, Phys. Rev. A 77, 042105 (2008).

[61] C. Bernhard, B. Bessire, A. Montina, M. Pfaffhauser, A. Stefanov, and S. Wolf, Non-Locality of Experimental Qutrit Pairs, J. Phys. A 47, 424013 (2014).

[62] H. S. Poh, S. K. Joshi, A. Cere, A. Cabello, and C. Kurtsiefer, Approaching Tsirelson's Bound in a Photon Pair Experiment, Phys. Rev. Lett. 115, 180408 (2015).

[63] C. Branciard, Detection Loophole in Bell Experiments: How Postselection Modifies the Requirements to Observe Nonlocality, Phys. Rev. A 83, 032123 (2011).

[64] S. L. Braunstein, A. Mann, and M. Revzen, Maximal Violation of Bell Inequalities for Mixed States, Phys. Rev. Lett. 68, 3259 (1992).

[65] B. Lang, T. Vértesi, and M. Navascués, Closed Sets of Correlations: Answers from the Zoo, J. Phys. A 47, 424029 (2014).

[66] J. Löfberg, in Proceedings of the CACSD Conference, Taipei, Taiwan, 2004. 